\title{
Reducing Dislocations of Antibiotic Hip Spacers via Hybrid Cement-screw Constrained Liner Fixation: A Case Series
}

\author{
Richard A. Pizzo, DO, Jay N. Patel, DO, Anthony Viola, DO, David M. Keller, DO, \\ Richard S. Yoon, MD, Frank A. Liporace, MD \\ Department of Orthopaedic Surgery, Jersey City Medical Center, Jersey City, NJ, USA
}

Purpose: Infection following total hip arthroplasty is a challenging and devastating complication. In two-stage revision arthroplasty, antibiotic spacers, although efficacious, can be associated with an unacceptable rate of mechanical complications (e.g., fracture, dislocation). This series describes 15 patients with infected total hip prostheses treated with hybrid cement-screw fixation constrained liner antibiotic spacers to enhance stability and minimize mechanical complications.

Materials and Methods: All patients with an infected hip prosthesis undergoing two-stage revision arthroplasty at a single academic medical center were identified and screened for inclusion. Clinical and radiographic data including patient demographics and outcome measures were collected and retrospectively analyzed.

Results: Two patients died of unrelated causes at an average of 6-week postoperatively. Infections in the remaining thirteen patients (100\%) were successfully eradicated; all underwent uncomplicated revision arthroplasty at a mean duration of 99.5 days after the placement of the antibiotic spacer. No dislocations, fractures, or other mechanical failures of any spacer were observed in this series.

Conclusion: The hybrid cement-screw fixation technique for constrained liner antibiotic spacers is a reliable and effective treatment method for eradicating prosthetic joint infections without mechanical complications.

Key Words: Arthroplasty, replacement, hip, Hip prosthesis, Prosthesis-related infections, Acetabulum, Polyethylene

Submitted: April 2, 2020 1st revision: April 23, 2020

Final acceptance: April 24, 2020

Address reprint request to

Richard S. Yoon, MD

(https://orcid.org/0000-0001-5240-6633)

Director, Orthopaedic Research, Division of Orthopaedic Trauma

\& Complex Adult Reconstruction, Department of Orthopaedic

Surgery, Jersey City Medical Center - RWJBarnabas Health, 377

Jersey Ave, Suite 280A, Jersey City, NJ 07302, USA

TEL: +1-201-716-5854 FAX: +1-201-309-2432

E-mail: yoonrichagmail.com

This is an Open Access article distributed under the terms of the Creative Commons Attribution Non-Commercial License (http://creativecommons. org/licenses/by-nc/4.0) which permits unrestricted non-commercial use, distribution, and reproduction in any medium, provided the original work is properly cited.

\section{INTRODUCTION}

Periprosthetic joint infection is a serious and dreaded complication following primary total hip arthroplasty (THA), occurring in $0.2 \%$ to $0.7 \%$ of primary THA and $0.95 \%$ to $22 \%$ of revision $\mathrm{THA}^{1-5)}$. The current gold standard for treating chronic periprosthetic infections in North America is a two-stage revision arthroplasty, which entails explant of the infected prosthesis, placement of a temporary antibiotic-laden cement spacer along with a course of intravenous antibiotics, and, finally, revision arthroplasty. The purpose of the antibiotic spacer is to provide a vehicle for local antibiotic administration that eradicates infection while simultaneously allowing for early joint mobilization and preser- 


\section{Hip \& Pelvis}

Hip Pelvis 32(4): 207-213, 2020

vation of joint space. Antibiotic spacers, however, are not without their own drawbacks, and complications can occur, particularly fracture or dislocation. Some studies have reported dislocation rates as high as $41 \%{ }^{6}$. Those who require surgical intervention for mechanical complication of their spacer have lower infection cure rates and a worse final clinical hip evaluation compared to patients without any mechanical complications ${ }^{7}$. Thus, minimizing mechanical complications while treating infected prostheses is essential to optimizing patient outcomes.

The purpose of this study was to analyze outcomes of a series of patients with infected hip prostheses treated with the fixed constrained liner antibiotic spacer technique to address acetabular bone loss and increase mechanical stability ${ }^{8}$.

\section{MATERIALS AND METHODS}

A single-institution total joint registry was screened retrospectively for inclusion. All patients diagnosed with periprosthetic THA infections treated surgically at a single academic medical center were identified. Inclusion criteria were as follows: i) patients with active prosthetic joint infections and ii) explant of infected prosthesis with place- ment of an antibiotic hip spacer using the hybrid screwcement fixed constrained liner technique. Prosthetic infections were diagnosed based on the criteria established by the Musculoskeletal Infection Society workgroup". Utilization of the cement-screw constrained liner technique was performed in all consecutive patients presenting to these senior authors with infected hip prostheses, and was verified utilizing operative notes and a review of intraoperative and postoperative radiographs. Patients were excluded if they: i) received spacers placed using other techniques, ii) had spacers placed for native hip septic arthritis or osteomyelitis, iii) had more than one concomitant prosthetic joint infection, and iv) had spacers implanted but to date have not yet reached the second stage of their treatment. No patients were excluded based on their medical comorbidities. After application of inclusion and exclusion criteria, 15 patients were identified for inclusion (Fig. 1). Data were collected using a predetermined data sheet via retrospective chart review, direct patient examination, and phone calls to patients and their families. Patient demographics, comorbidities, infectious workup results, operative data, and clinical and radiographic follow-up information were recorded. Institutional review board (IRB) approval was obtained prior to the start of the study (Jersey City Medical Center;

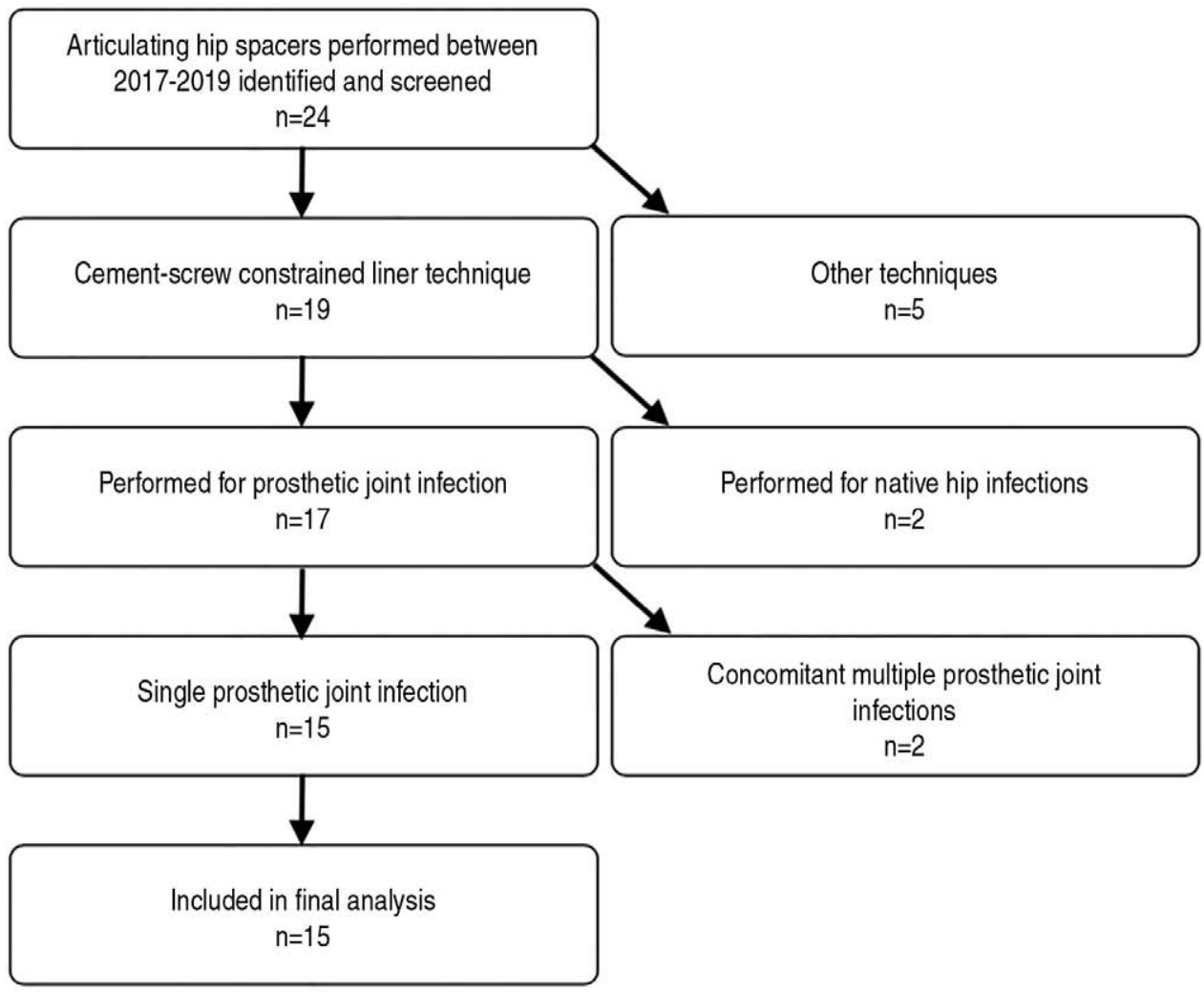

Fig. 1. Inclusion and exclusion criteria for patient identification and selection. 


\section{Hip \& Pelvis}

Richard A. Pizzo et al. Reducing Dislocations of Antibiotic Hip Spacers via Hybrid Cement-screw Constrained Liner Fixation

IRB No. 20171537). Statistical analysis was performed using Microsoft Excel (Microsoft Inc, Redmond, WA, USA).

The hip spacer technique employed was the hybrid screwcement fixed constrained liner technique described by Patel et al. ${ }^{8}$ through a posterior approach. For this technique, a constrained liner is cemented into the acetabulum and fixed with a countersunk screw through the liner and into the ilium after explant of the infected prosthesis. A true femoral head component is mounted onto a custom-molded antibiotic spacer stem to form the femoral component (Fig. 2). The antibiotic cement utilized included $3 \mathrm{~g}$ powdered vancomycin and $3.6 \mathrm{~g}$ powdered tobramycin per bag of cement. Numerous cultures and frozen sections were obtained during each procedure. All spacers were implanted by one of two primary surgeons at a single academic medical center. Treatment courses for all patients followed the algorithm shown in
Fig. 3. After explant of infected prosthesis and insertion of antibiotic cement spacer, all patients received a minimum of 6 weeks of intravenous antibiotics followed by a 2- to 4-week antibiotic holiday, after which new infectious labs consisting of white blood cell count, erythrocyte sedimentation rate, C-reactive protein were drawn before proceeding with the second stage definitive revision arthroplasty or inserting a second antibiotic spacer. Decisions on when to proceed with the second-stage procedure were based on likelihood of infection eradication as determined by an infectious disease specialist as informed by inflammatory markers and compliance with follow-up. Frozen sections were obtained and analyzed for number of polymorphonuclear cells (PMNs) per high-power-field (HPF) at all second-stage procedures. Patients were considered to be eradicated of infection if they exhibited normal or significantly down-trend-
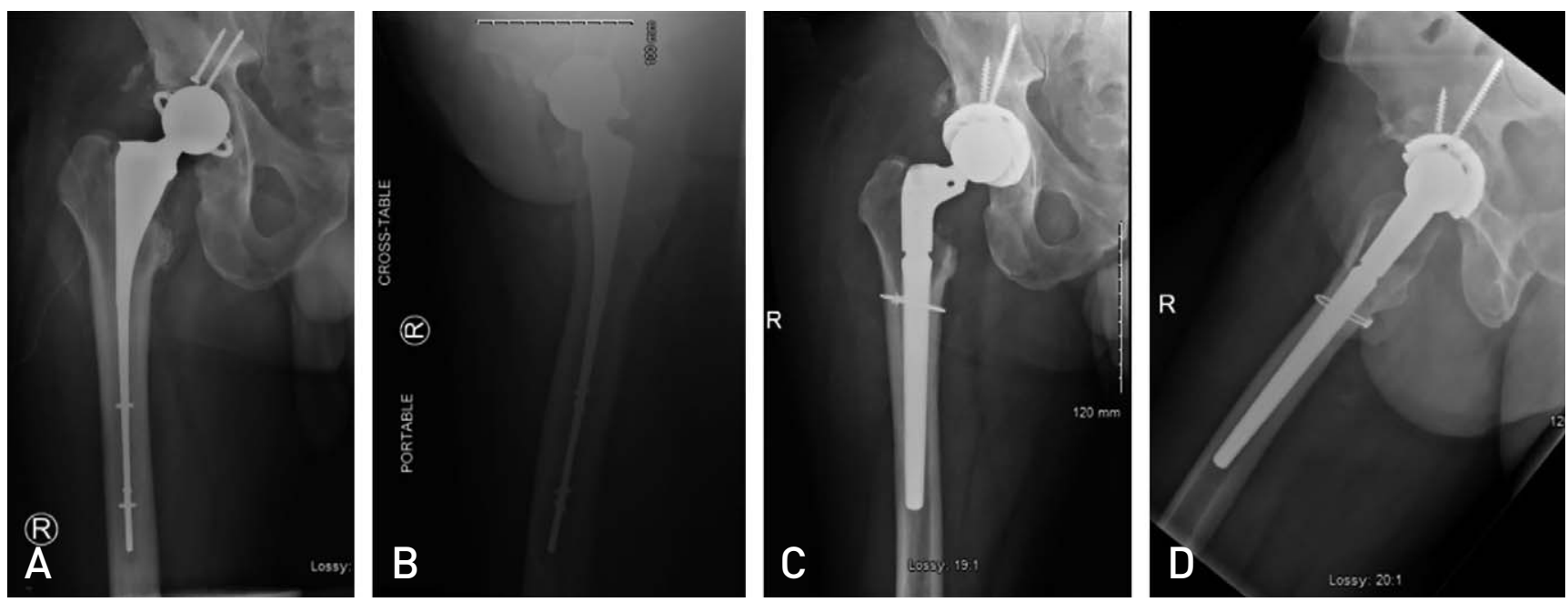

Fig. 2. Anteroposterior (A) and lateral (B) radiographs of the hybrid cement-screw fixed constrained liner antibiotic spacer construct. Anteroposterior (C) and lateral (D) radiographs of the same patient status post revision arthroplasty.

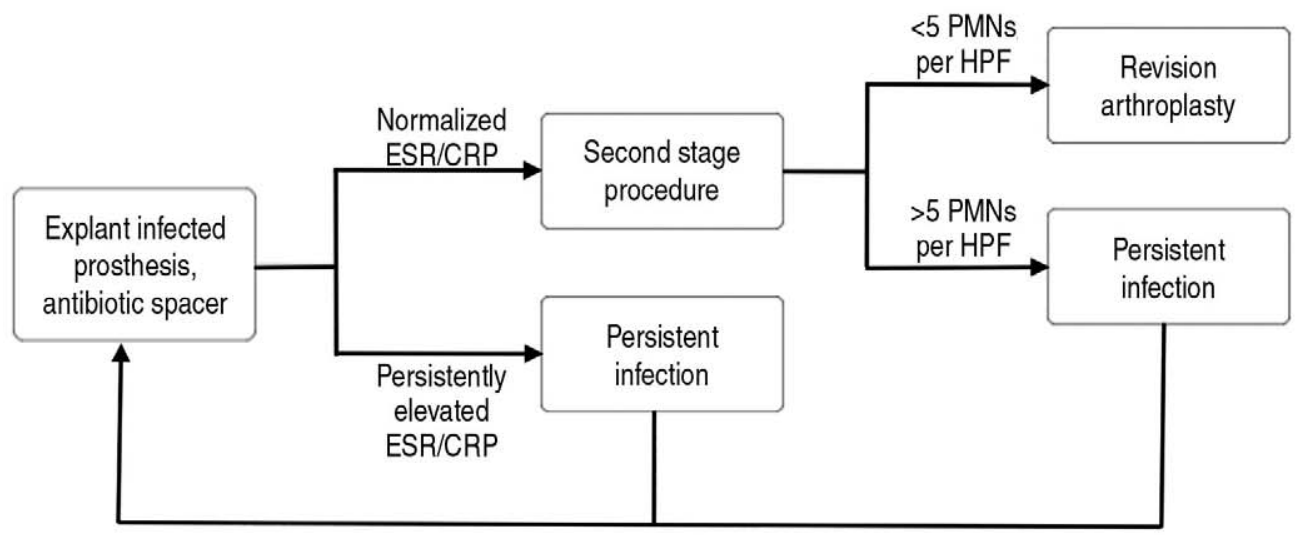

Fig. 3. Treatment algorithm for infected hip prostheses utilized in this series.

ESR: erythrocyte sedimentation rate, CRP: C-reactive protein, PMN: polymorphonuclear cell, HPF: high-power-field. 
ing inflammatory markers at 8-week after spacer implantation and demonstrated $<5$ PMNs per HPF on intraoperative frozen section at time of second-stage procedure. These patients underwent revision arthroplasty either with revision components or proximal femur replacement, depending on intraoperative assessment of the remaining bone stock. Failures of eradication were defined as persistently elevated or up-trending inflammatory markers after twelve weeks of spacer implantation, or patients with $>5$ PMNs per HPF intraoperatively during the second stage procedure. These patients underwent placement of a second antibiotic spacer (Fig. 3).

\section{RESULTS}

A total of 15 spacers were implanted in 15 patients (11 males and 4 females), with mean age of $64.5 \pm 13.7$ years at time of spacer implantation (Table 1). Five patients had

Table 1. Demographic and Clinical Outcomes

\begin{tabular}{lc}
\hline \hline Variable & Value \\
\hline Mean age (yr) & $64.5 \pm 13.7$ \\
Sex & $11(73.3)$ \\
Male & $4(26.7)$ \\
Female & \\
Comorbidities & $5(33.3)$ \\
Tobacco use & $2(13.3)$ \\
HIV & $2(13.3)$ \\
Hepatitis C & $1(6.7)$ \\
ESRD on hemodialysis & $1(6.7)$ \\
Sickle cell anemia & \\
Culture results & $7(46.7)$ \\
Staphylococcal spp. & $1(6.7)$ \\
Streptococcus spp. & $3(20.0)$ \\
Gram negative & $2(13.3)$ \\
Polymicrobial & $2(13.3)$ \\
No organism isolated & $99.5 \pm 31.9$ \\
Mean spacer duration (day) & \\
Definitive procedure & $11(84.6)$ \\
Revision THA & $2(15.4)$ \\
Proximal femur replacement & $1(7.7)$ \\
Complications & $1(7.7)$ \\
Reinfection & $1(7.7)$ \\
Wound complication & \\
Reoperations & \\
Irrigation and debridement, & \\
polyethylene exchange & \\
\hline
\end{tabular}

Values are presented as mean \pm standard deviation or number $(\%)$.

HIV: human immunodeficiency virus, ESRD: end stage renal disease, THA: total hip arthroplasty. at least one attempted revision arthroplasty prior to presenting to one of the senior authors. Mean follow-up from time of spacer implantation was $56.0 \pm 28.7$ weeks. Five of the 15 patients (33.3\%) were smokers. Three of the included patients were immunocompromised (human immunodeficiency virus [HIV] $[n=1]$; hepatitis $\mathrm{C}[\mathrm{n}=1]$; HIV, hepatitis $\mathrm{C}$, and end-stage renal disease on hemodialysis $[n=1])$. Two of the 15 patients died with antibiotic spacers in place at an average of 6-week postoperatively. One patient died of pulmonary complications secondary to a sickle cell crisis at an outside hospital. The final patient suffered a cardiac arrest while at dialysis; medical history for this patient was significant for HIV, chronic kidney disease and liver cirrhosis, and with a poor prognosis of neurologic recovery, the family decided to discontinue care. Of the remaining patients, all $13(100 \%)$ had successful eradication of their periprosthetic infection and underwent successful second-stage revision arthroplasty. Eleven of these patients were revised to modular diaphyseal-fitting revision components with constrained liners, augmented by trochanteric fixation when necessary, and two underwent proximal femur replacement. Average duration of spacer implantation was $99.5 \pm 31.9$ days. While spacers were in place, 6 patients were made non-weight bearing, 5 toe-touch weight bearing, 2 partial weight bearing, and 2 weight bearing as tolerated. Culture results at time of explantation yielded methicillin-resistant Staphylococcus aureus in 4 patients (26.7\%), methicillin-sensitive Staphylococcus aureus in 3 patients (20.0\%), and gram-negative organisms in 3 patients $(20.0 \%)$. Two patients exhibited polymicrobial culture growth; no specific bacteria were isolated from 2 patients. There were no hip dislocations, fractures about spacers, or other mechanical complication to any spacer observed in this series.

There was one case of reinfection, which required an additional procedure. Importantly, this patient had a long history of multiply infected arthroplasty and undergone a number of surgeries prior to presenting to the senior author. After an uneventful spacer course, he was deemed clear of infection and underwent revision arthroplasty. Postoperatively, he was noncompliant with his posterior hip precautions and toe-touch weightbearing restrictions; furthermore, he sustained a prosthetic hip dislocation that was successfully corrected via closed reduction. Additionally, this patient never followed-up with an infectious disease specialist as requested and was ultimately lost to follow-up for a time, until he presented 6-month later complaining of a draining wound over his surgical incision. Ultimately, it was suggest- 
Richard A. Pizzo et al. Reducing Dislocations of Antibiotic Hip Spacers via Hybrid Cement-screw Constrained Liner Fixation

ed that this individual undergo a repeat two-stage revision arthroplasty procedure with antibiotic cement spacer, however he refused and ultimately only agreed to undergo a single-stage irrigation and debridement with polyethylene exchange. He was doing well at final clinical follow-up with no further evidence of infection.

All remaining patients had uncomplicated postoperative courses, with no clinical, radiographic, or laboratory findings of reinfection of their revision prosthesis, and no dislocations or mechanical complications.

\section{DISCUSSION}

Two-stage revision arthroplasty utilizing an antibiotic spacer is the current gold standard for the treatment of prosthetic joint infections in North America. Though efficacious, hip antibiotic spacers have historically been associated with high rates of mechanical complications, namely spacer dislocation and fracture, and many spacer techniques have been described to minimize these risks. This study describes a series of patients treated with a hybrid cementscrew constrained liner spacer technique with an overall infection eradication rate of $93 \%$ and zero mechanical failures.

Prosthetic joint infection is a serious and potentially devastating complication of arthroplasty procedures. Though relatively infrequent -occurring in $0.2 \%$ to $0.7 \%$ of primary THA and $0.95 \%$ to $22 \%$ of revision THA $^{1-5)}$, - the incidence of prosthetic joint infection is increasing at a significant rate ${ }^{10)}$. Current treatment strategies with antibiotic spacers confer substantial psychological and physical burden on patients, entailing at least two additional major operations with corresponding physical limitations and the longterm use of potentially noxious antibiotics. Despite this aggressive approach, up to $35 \%$ of patients are unable to clear their infections and may require a multitude of salvage procedures (e.g., fusion, resection arthroplasty, amputation) $)^{11-14)}$. While the effects of these grave salvage procedures on the individual are presumably morbid, the burden to society is also great. In the United States, recent data estimates that the cost of treating prosthetic joint will reach $\$ 1.62$ billion by $2020^{15)}$. Parisi and colleagues ${ }^{16)}$ estimated that the lifetime cost of treating an infected THA in a 65-year-old is $\$ 390,806$. In their analysis, indirect costs such as lost wages made up a large portion of the costs. Due to the gravity of both the economic and personal burden on patients, and also in light of the increasing incidence of prosthetic joint infection as more arthroplasty procedures are performed in North America, it is important to optimize treatments to limit morbidity and mortality $^{10)}$.

Several different techniques are available for implantation of antibiotic hip spacers. Broadly, spacers may be categorized as static or mobile. A spacer is considered static if it does not allow for range of motion exercises or partial weight bearing. In this scenario, the infections on the acetabular and femoral sides are addressed with independent constructs that are not intended to articulate in a physiologic manner, and the hip joint is left in discontinuity. A mobile, or articulating, spacer is any construct in which the acetabular and femoral components communicate in a way that allows for relatively physiologic motion of the hip ${ }^{17)}$. The advantages of mobile hip spacers include early joint mobilization and the potential for weight bearing through the construct $^{17-19)}$. Mobile spacers may be handmade, custommolded, prefabricated, or metal-on-polyethylene. Relatively few studies exist comparing these various techniques, however, and little consensus exists regarding optimal surgical technique.

Antibiotic spacers have historically been associated with unacceptably high rates of mechanical complications. The most common mechanical complications associated with antibiotic spacers are fracture and dislocation. Reported rates of spacer dislocation range widely from $0 \%$ to $41 \%{ }^{6,17,20-23)}$. Those who require surgical intervention for mechanical complication of their spacer have lower infection cure rate and worse functional outcome scores, therefore, minimizing mechanical complications while treating infected prostheses is essential to optimizing patient outcomes ${ }^{7}$.

The increased stability and lack of mechanical failure of the fixed constrained liner construct utilized in this series is likely multifactorial in nature. Two factors known to contribute to spacer instability are acetabular bone loss and patient inability to comply with non-weight bearing restrictions ${ }^{17,24,25)}$. Acetabular bone loss in the setting of prosthetic joint infection can occur due to osteoclast-mediated osteolysis in response to inflammatory cytokines, or iatrogenically during difficult acetabular component remova ${ }^{17,26)}$. The spacer technique performed in this series helps to address these bony deficits by utilizing a rebar cement-screw mantle construct to recreate the normal architecture of the acetabulum, which eliminates any potential instability related to issues of acetabular component fixation or uncoverage. Additionally, the constrained liner utilized in this series also confers significant stability to this construct. Use of constrained liners has been shown to be highly successful in preventing prosthetic hip dislocation in patients at high 
risk for recurrent instability ${ }^{27}$. Finally, the true articulating nature of this antibiotic spacer technique allows the potential for immediate weight bearing, which obviates the need for strict adherence to postoperative non-weight bearing precautions. Similar articulating spacers have been shown to be associated with better functional hip scores and better walking capacity, less blood loss during revision surgery, and fewer postoperative dislocation ${ }^{23}$. Nine of the 15 patients $(60.0 \%)$ were permitted at least partial weight bearing while their spacers were in place. These factors all likely contributed to the zero mechanical failures observed in this series.

In addition to experiencing no mechanical complications, the patients in this series had favorable outcomes overall. All thirteen patients (100\%) successfully cleared their infections, as evidenced by normalized infectious laboratory markers and intraoperative cultures and frozen sections. Though there was one case of reinfection that was successfully treated with an additional procedure, this eradication rate is comparable or superior to existing literature (i.e., $65-90 \%)^{11-14)}$. All patients in this series underwent successful secondstage revision arthroplasty procedures over the course of this study and were doing well at final clinic follow-ups.

\section{CONCLUSION}

As the number of infected THA increases, it is important to develop techniques that can reliably treat infection while minimizing morbidity and mortality. The hybrid cementscrew fixation for constrained liner antibiotic spacers is a reliable and effective treatment method for eradicating prosthetic joint infections without mechanical complications.

\section{CONFLICT OF INTEREST}

The authors declare that there is no potential conflict of interest relevant to this article.

\section{REFERENCES}

1. Mahomed NN, Barrett JA, Katz JN, et al. Rates and outcomes of primary and revision total hip replacement in the United States medicare population. J Bone Joint Surg Am. 2003; 85:27-32.

2. Phillips CB, Barrett JA, Losina E, et al. Incidence rates of dislocation, pulmonary embolism, and deep infection during the first six months after elective total hip replacement. J Bone Joint Surg Am. 2003;85:20-6.

3.Zhan C, Kaczmarek R, Loyo-Berrios N, Sangl J, Bright RA. Incidence and short-term outcomes of primary and revision hip replacement in the United States. J Bone Joint Surg Am.
2007;89:526-33.

4. Garvin KL, Hanssen AD. Infection after total hip arthroplasty. Past, present, and future. J Bone Joint Surg Am. 1995;77:157688.

5. Blom AW, Taylor AH, Pattison G, Whitehouse S, Bannister GC. Infection after total hip arthroplasty. The Avon experience. J Bone Joint Surg Br. 2003;85:956-9.

6. Leunig M, Chosa E, Speck M, Ganz R. A cement spacer for two-stage revision of infected implants of the hip joint. Int Orthop. 1998;22:209-14.

7. Bori G, García-Oltra E, Soriano A, Rios J, Gallart X, Garcia S. Dislocation of preformed antibiotic-loaded cement spacers (Spacer-G): etiological factors and clinical prognosis. $J$ Arthroplasty. 2014;29:883-8.

8. Patel JN, Pizzo RA, Yoon RS, Liporace FA. Addressing antibiotic hip spacer instability via hybrid screw-cement fixation of a constrained liner and cement-rebar interface techniques: a technical narrative. J Am Acad Orthop Surg. 2020;28:16670.

9. Parvizi J, Zmistowski B, Berbari EF, et al. New definition for periprosthetic joint infection: from the Workgroup of the Musculoskeletal Infection Society. Clin Orthop Relat Res. 2011;469:2992-4.

10. Kurtz SM, Lau E, Schmier J, Ong KL, Zhao K, Parvizi J. Infection burden for hip and knee arthroplasty in the United States. J Arthroplasty. 2008;23:984-91.

11. Aggarwal VK, Rasouli MR, Parvizi J. Periprosthetic joint infection: current concept. Indian J Orthop. 2013;47:10-7.

12. Babis GC, Sakellariou VI, Pantos PG, Sasalos GG, Stavropoulos NA. Two-stage revision protocol in multidrug resistant periprosthetic infection following total hip arthroplasty using a long interval between stages. J Arthroplasty. 2015;30:1602-6.

13. Senthi S, Munro JT, Pitto RP. Infection in total hip replacement: meta-analysis. Int Orthop. 2011;35:253-60.

14. Parvizi J, Zmistowski B, Adeli B. Periprosthetic joint infection: treatment options. Orthopedics. 2010;33:659.

15. Kurtz SM, Lau E, Watson H, Schmier JK, Parvizi J. Economic burden of periprosthetic joint infection in the United States. J Arthroplasty. 2012;27(8 Suppl):61-5.e1.

16. Parisi TJ, Konopka JF, Bedair HS. What is the long-term economic societal effect of periprosthetic infections after THA? A Markov analysis. Clin Orthop Relat Res. 2017;475:1891900.

17. Jacobs C, Christensen CP, Berend ME. Static and mobile antibiotic-impregnated cement spacers for the management of prosthetic joint infection. J Am Acad Orthop Surg. 2009; 17:356-68.

18. Anagnostakos K, Jung J, Schmid NV, Schmitt E, Kelm J. Mechanical complications and reconstruction strategies at the site of hip spacer implantation. Int J Med Sci. 2009;6: 274-9.

19. Evans RP. Successful treatment of total hip and knee infection with articulating antibiotic components: a modified treatment method. Clin Orthop Relat Res. 2004;(427):37-46.

20. Koo KH, Yang JW, Cho SH, et al. Impregnation of vancomycin, gentamicin, and cefotaxime in a cement spacer for two-stage cementless reconstruction in infected total hip arthroplasty. J Arthroplasty. 2001;16:882-92.

21. Romanò CL, Romanò D, Logoluso N, Meani E. Long-stem 


\section{Hip \& Pelvis}

Richard A. Pizzo et al. Reducing Dislocations of Antibiotic Hip Spacers via Hybrid Cement-screw Constrained Liner Fixation

versus short-stem preformed antibiotic-loaded cement spacers for two-stage revision of infected total hip arthroplasty. Hip Int. 2010;20:26-33.

22. Faschingbauer M, Reichel H, Bieger R, Kappe T. Mechanical complications with one hundred and thirty eight (antibioticladen) cement spacers in the treatment of periprosthetic infection after total hip arthroplasty. Int Orthop. 2015;39:989-94.

23. Hsieh PH, Shih CH, Chang YH, Lee MS, Shih HN, Yang WE. Two-stage revision hip arthroplasty for infection: comparison between the interim use of antibiotic-loaded cement beads and a spacer prosthesis. J Bone Joint Surg Am. 2004;86:198997.
24. Anagnostakos K, Fürst O, Kelm J. Antibiotic-impregnated PMMA hip spacers: current status. Acta Orthop. 2006;77: 628-37.

25. Citak M, Masri BA, Springer B, Argenson JN, Kendoff DO. Are preformed articulating spacers superior to surgeon-made articulating spacers in the treatment of PJI in THA? A literature review. Open Orthop J. 2015;9:255-61.

26. Mbalaviele G, Novack DV, Schett G, Teitelbaum SL. Inflammatory osteolysis: a conspiracy against bone. J Clin Invest. 2017;127:2030-9.

27. Su EP, Pellicci PM. The role of constrained liners in total hip arthroplasty. Clin Orthop Relat Res. 2004;(420):122-9. 